\title{
Chronic gastrointestinal problems and bowel dysfunction in patients with spinal cord injury
}

\author{
Tai Ryoon Han, Jin Ho Kim and Bum Sun Kwon \\ Department of Rehabilitation Medicine, Seoul National University College of Medicine, 28 Yoengun-dong, \\ Chongro-ku, Seoul, Korea
}

\begin{abstract}
Amongst complications arising from spinal cord injury (SCI), chronic gastointestinal (G-I) problems and bowel dysfunction have not received as much research attention as many other medical and rehabilitation problems, even although their incidence is not negligible. We therefore investigated chronic G-I problems and bowel dysfunction in SCI patients where the degree of these was such that activities of daily living (ADL) were significantly affected and/or long-term medical management was required. Detailed semi-structured individual interviews were conducted with 72 traumatic SCI patients. The history of SCI was longer than 6 months, bowel habits had settled, and neurological recovery was completed. The incidence of chronic G-I problems was very high $(62.5 \%)$, most were associated with defecation difficulties such as severe constipation, difficult with evacuation, pain associated with defecation, or urgency with incontinence. These problems had an extensive impact on ADL, and in particular, restricted diet $(80 \%)$, restricted outdoor ambulation $(64 \%)$ and caused unhappiness with bowel care $(62 \%)$. Bowel care was performed once per $2.85 \pm 1.96$ days and occupied an average of $42.1 \pm 28.7 \mathrm{~min}$. To improve bowel habits, $43 \%$ of the patients took oral medication, and $36.1 \%$ controlled their diet. The usual methods of bowel care were anal massage $(34.7 \%)$, unaided self-defecation with or without oral medication and abdominal massage (29.2\%), finger enema $(18.1 \%)$, rectal suppository $(15.2 \%)$ and in two patients a colostomy tube had been inserted because of rectal cancer and traumatic colorectal injury. These chronic G-I symptoms were vague and very subjective, but significant enough to affect the quality of life. Bowel dysfunction was not related to age, duration of, or the neurological level of injury, ASIA score of ADL level, and bowel habits had generally settled within 6 months of SCI. With regard to frequency, time, and method of defecation, bowel care habits varied considerably amongst individuals, and in relation to the extent to which practical results matched the level of expectation generated by a physicians' recommended care program. Individual satisfaction was also very subjective. We therefore suggest that during the early stage of rehabilitation, an appropriate bowel program should be properly designed and adequate training provided.
\end{abstract}

Keywords: spinal cord injury; neuropathic bowel; gastrointestinal problems; bowel dysfunction; activity of daily living

\section{Introduction}

With the control of urological and cardiopulmonary complications in SCI patients and the introduction of rehabilitation, the life span of such patients has increased and more have been able to return to work. $^{1,2}$ A current goal of SCI rehabilitation should therefore be to improve the quality of life; to this end, the improvement of bowel function in patients with chronic G-I problems and bowel dysfunction would

Correspondence: Dr Tai Ryoon Han, MD, PhD, Professor and Chairman of Department of Rehabilitation Medicine, Seoul National University College of Medicine, 28 Yoengun-dong, Chongro-ku, Seoul, 100-744, Korea help considerably. Most research related to such problems has focused on the 'acute abdomen' developed immediately after SCI or an operation for $\mathrm{it}^{3-6}$ and prophylatic management, such as the administration of $\mathrm{H} 2$ inhibitor for stress-related ulcer, reduced the incidence of G-I problems. ${ }^{2}$ These are not uncommon even in healthy population, ${ }^{7}$ however, and may be chronic, and it may therefore be suspected that they are also common among SCI patients have a great impact on ADL. Several studies have addressed chronic G-I problems and bowel dysfunction, but its characteristics and impact on the activity of daily living (ADL) have not yet been widely studied. We therefore investigated the characteristics of chronic G-I problems 
and bowel dysfunction, where the degree of these was such that daily activities (ADL) were significantly affected or long-term medical management was required. We also determined which factors affect bowel dysfunction.

\section{Materials and methods}

\section{Patients}

Traumatic SCI patients, including those involved in traffic accidents or falls, participated in this study; at least 6 months had elapsed since the SCI, and they had already completed primary care, having undergone an operation or been subject to conservative management. Each participant was either an in-patient at Seoul National University Hospital, who had been newly admitted there for evaluation of kidney function, was an in-patient at the National Rehabilitation Center, who had vocational therapy or secondary care after discharge from a primary care hospital, or was receiving nursing care at a community society, Eumsung Kkottongne, Chung-buk province, Korea. The patients with a combined cauda equina lesion were excluded, and also excluded were those with a spinal cord lesion due to disease, such as tumor, or as a sequel of brain damage at the time of the SCI; also excluded were those whose neurological recovery was ongoing or whose bowel habit unsettled.

\section{Method}

Interviews were performed individually; their format was semi-structured and they included questions relating to G-I problems significant enough to have adverse impact on ADL or require long-term management, the kind of impact, and the characteristics of bowel care habits. Physical examination was performed and the neurological level of SCI was evaluated as tetraplegic $(\mathrm{C} 4-\mathrm{C} 8)$, high paraplegic $(\mathrm{T} 1-\mathrm{T} 6)$, or low paraplegic (below T7), and severity of injury was evaluated as 'A', 'B', 'C' or 'D' according to ASIA Impairment scale (' $\mathrm{E}$ ' is not included on this study). Medical records detailing current medication and specific management for G-I problems were reviewed when available; patients without such records supplied relevant details of their medical history.

Daily activity (ADL) was evaluated using the Modified Barthel Index score, and patients were grouped according to whether they were totally dependent (a score of 20 or less), severely dependent $(21-61)$, moderately dependent $(62-90)$, or independent (91 or more). ${ }^{8}$ Actual current activity was simultaneously evaluated according to the ambulation level and patients were assigned to one of three groups; 'community ambulation' group, 'household ambulation' group including those who ambulated in the hospital only, and 'bed-confinement' group (those of older age, cardiopulmonary dysfunction, or severe lower extremity contracture, for example).

\section{Statistics}

Patients were categorized according to age, duration and neurological level of SCI, ASIA scale, degree of dependency of ADL level and ambulatory activity level. Whether or not these tended to influence bowel dysfunction was determined from the results of the Mantel-Haenszel chi-test, using the SAS analysis system. The Chi-test was also used to analyze characteristics of bowel habits, whether these were closely related to bowel dysfunction.

\section{Results}

\section{Characteristics of chronic G-I problems and their incidence}

Seventy-two patients voluntarily participated in this series by interviews; 48 males and 24 females. The average age was $38.0 \pm 11.9$ years. Mean duration of spinal cord injury was $37.5 \pm 45.2$ months. Forty-five of these $72(62.5 \%)$ reported chronic G-I problems, significant enough to have an adverse impact on the ADL or requiring long-term management. Severe constipation was the commonest problem, affecting $43.1 \%$ of patients, while difficulty with evacuation $(33.3 \%)$ and post-prandial discomfort $(33.3 \%)$ were the next most frequent. Other problems were vague abdominal discomfort (25\%), gastrointestinal reflux symptoms, which were very vague and non-specific $(15.3 \%)$, incontinence due to urgency $(13.9 \%)$, and autonomic dysreflexia associated with gastrointestinal symptoms $(8.3 \%)$ (Table 1$)$.

The impact of these chronic G-I problems on ADL was categorized as follows. First, 36 patients $(80 \%)$ complained of 'restriction of diet', which was that abdominal distension or discomfort at the time of defecation caused fear of eating, and severe constipation which always followed diarrhea made menu selection problem. Second, 29 patients (64.4\%) complained of 'restriction of outdoor ambulation',

Table 1 Chronic G-I problems, significant enough to impact on $\mathrm{ADL}$ or require long-term management

\begin{tabular}{lcc}
\hline Problems & No. of cases & $\%$ \\
\hline Severe constipation & 31 & 43.1 \\
Difficulty with evacuation & 24 & 33.3 \\
Post-prandial discomfort & 24 & 33.3 \\
Vague abdominal pain & 18 & 25.0 \\
Pain associated with defecation & 13 & 18.1 \\
Gastrointestinal reflux symptom & 11 & 15.3 \\
$\quad$ including nausea & 11 & 15.3 \\
Bleeding after defecation including & & \\
$\quad$ hemorrhoid & 10 & 13.9 \\
Urgency and incontinence & 6 & 8.3 \\
Autonomic dysreflexia G-I origin & & \\
Chronic G-I problems* & 45 & 62.5 \\
\hline
\end{tabular}

*One G-I problem or more 
which was that increased activity outdoor often induced the unexpected need to defecate and it was hard to solve. Twenty-two patients $(48.8 \%)$ complained of 'difficulty and unhappiness of bowel care' and they believed that without such difficulties, their quality of life would see great improvement.

Before their accident (pre-SCI), 15 patients had G-I problems, and all of these patients had aggravation of such problems after the accident (post-SCI). Also, other problems, such as urgency and autonomic dysreflexia, developed only post-SCI (Table 2). Chronic G-I problems newly developed post-SCI were thus mostly associated with defecation difficulty, which included severe constipation (26 patients), difficulty with evacuation (24), pain associated with defecation (9) and urgency accompanied by incontinence (9). We can therefore state that post-SCI gastrointestinal problems were mainly bowel dysfunction, that is defecation difficulty $(40.3 \%)$, and these problems markedly affected ADL.

\section{Characteristics of bowel habit in SCI patients}

Defecation was performed on an average once per $2.85 \pm 1.96$ days. The total time involved, from the moment of deciding to initiate the process of defecation, and then transfer to the toilet, to completion, was $42.1 \pm 28.7 \mathrm{~min}$; for evacuation only, ie from stimulation to completion, was $24.7+12.9 \mathrm{~min}$, provided by only 30 patients. To help defecation, 30 of 72 patients $(41.7 \%)$ had always used oral medication, namely G-I motility drug and laxative, and $25(34.7 \%)$ controlled their diet. In 52 of 72 patients $(72.2 \%)$, bowel habit had settled within 6 months of SCI, the early stage of rehabilitation and in most patients $(93.1 \%)$, these habits had settled within 1 year (Table $3)$.

Table 2 Chronic G-I problems developed post-SCI

\begin{tabular}{|c|c|c|c|}
\hline G-I problems & Pre-SCI & $\begin{array}{l}\text { Post-SCI } \\
\text { worsening }\end{array}$ & $\begin{array}{c}\text { Post-SCI } \\
\text { newly } \\
\text { developed }\end{array}$ \\
\hline Severe constipation* & 5 & 5 & 26 \\
\hline Difficulty with evacuation* & 0 & 0 & 24 \\
\hline post-prandial discomfort & 8 & 0 & 16 \\
\hline vague abdominal discomfort & 12 & 12 & 6 \\
\hline $\begin{array}{l}\text { Pain associated with } \\
\text { defecation* }\end{array}$ & 2 & 2 & 9 \\
\hline $\begin{array}{l}\text { gastrointestinal reflux } \\
\text { symptom }\end{array}$ & 5 & 5 & 6 \\
\hline $\begin{array}{l}\text { bleeding after defecation } \\
\text { including hemorrhoid }\end{array}$ & 2 & 2 & 9 \\
\hline Urgency and incontinence* & 0 & 0 & 9 \\
\hline $\begin{array}{l}\text { autonomic dysreflexia } \\
\text { G-I origin }\end{array}$ & 0 & 0 & 6 \\
\hline Defecation difficulty* & & \multicolumn{2}{|c|}{$40.3 \%(29 / 72)$} \\
\hline
\end{tabular}

Numbers are cases
For 25 patients (34.7\%), the usual method for bowel care was anal massage for reflex defecation, and 21 patients $(29.2 \%)$ managed unaided self-defecation with or without oral medication and abdominal massage. Thirteen patients $(18.1 \%)$ used a finger enema, and 11 $(15.2 \%)$ used a rectal suppository. Two patients had had an accidental colostomy; in one, this was due to rectal cancer, and in the other was a traumatic colorectal injury at the time of SCI (Table 4).

\section{Factors affecting bowel dysfunction}

To determine which factors affect bowel dysfunction, age, duration and level of SCI, ASIA score, level of dependency in ADL and ambulatory activity level were investigated, but none of these factors were statistically significant (Table 5). Because of the small number in the bed-confinement group, the statistical significance of ambulatory activity level was not significant enough to affect the bowel dysfunction, even although the colostomy case was excluded.

Of the three factors involved in bowel care habit, namely bowel care method, frequency and time, patients using self-controlled reflex defecation, with or without oral medication, had less defecation difficulty than did those using mechanical aids such as a finger enema or a rectal suppository. Regarding the frequency and time for defecation, patients whose intervals were short, and completed defecation within a short time, experienced less difficulty, whilst all patients whose

Table 3 Characteristics of bowel care habit in SCI patients

\begin{tabular}{lc}
\hline Frequency & once per $2.85 \pm 1.96$ \\
days & $42.1 \pm 28.7 \mathrm{~min}$ \\
Time for defecation, total bowel care & $24.7 \pm 12.9 \mathrm{~min}$ \\
evacuation only* & $41.7 \%(30 / 72)$ \\
Oral medication for defecation & $34.7 \%(25 / 72)$ \\
Diet control for defecation & $10.2 \pm 17.3$ months \\
Time for bowel habit settled & $72.2 \%(52 / 72)$ \\
within 6 months & $20.8 \%(15 / 72)$ \\
between 6 and 12 months & $6.9 \%(5 / 72)$ \\
over 12 months & \\
\hline
\end{tabular}

( ); numbers are cases/total. *For only 30 cases

Table 4 Usual bowel care method

\begin{tabular}{lcc}
\hline Method & $\begin{array}{c}\text { Number } \\
\text { of cases }\end{array}$ & $\%$ \\
\hline Anal massage & 25 & 34.7 \\
Self defecation $^{\text {a }}$ & 21 & 29.2 \\
Finger enema $_{\text {Rectal suppository }}$ & 13 & 18.1 \\
Colostomy $^{\text {b }}$ & 11 & 15.2 \\
\hline
\end{tabular}

${ }^{a}$ Unaided self defecation with or without oral medication and abdominal massage. ${ }^{b}$ Rectal cancer and traumatic colorectal injury 
defecation time was more than 60 min complained of defecation difficulty. However half the patients with a defecation interval of over 5 days reported no difficulties at all, since most had controlled their bowel frequency for longer because of unhappiness and the time consumed by bowel care. It was also noticeable that two of 12 patients, whose defecation time was brief, below $20 \mathrm{~min}$ had complained of difficulty with evacuation, a kind of defecation difficulty (Table 6). These findings suggest that bowel dysfunction is very subjective and that in bowel care, the concept of 'satisfactory' varied considerably among individuals.

\section{Ideal bowel care, Physicians vs Patients}

The instruction of most physicians is that bowel care should be self-controlled and spontaneous, with or without oral medication, and performed at least once every 2 days and within $30 \mathrm{~min}$; this, according to physicians, is 'ideal bowel care of physician'. The bowel

Table 5 Effect of various factors on bowel dysfunction

\begin{tabular}{|c|c|c|c|}
\hline \multirow[b]{2}{*}{ Factors } & \multicolumn{2}{|c|}{ Defecation difficulty } & \multirow[b]{2}{*}{$P$-value } \\
\hline & Present & Absent & \\
\hline \multicolumn{4}{|l|}{ Age (year) } \\
\hline below 30 & 9 & 17 & NS \\
\hline $30-40$ & 11 & 8 & \\
\hline $41-50$ & 4 & 8 & \\
\hline $51-60$ & 5 & 7 & \\
\hline above 60 & 0 & 7 & \\
\hline \multicolumn{4}{|l|}{ SCI duration (months) } \\
\hline below 9 & 4 & 6 & NS \\
\hline $9-12$ & 3 & 7 & \\
\hline $13-24$ & 11 & 12 & \\
\hline $25-48$ & 4 & 10 & \\
\hline above 48 & 7 & 8 & \\
\hline \multicolumn{4}{|l|}{ SCI level } \\
\hline $\mathrm{C} 4-\mathrm{C} 8$ & 11 & 15 & NS \\
\hline $\mathrm{T} 1-\mathrm{T} 6$ & 7 & 13 & \\
\hline below T6 & 11 & 15 & \\
\hline \multicolumn{4}{|l|}{ ASIA scale } \\
\hline A & 16 & 16 & NS \\
\hline B & 5 & 10 & \\
\hline $\mathrm{C}$ & 5 & 15 & \\
\hline $\mathrm{D}$ & 3 & 2 & \\
\hline \multicolumn{4}{|c|}{ Dependency of ADL level } \\
\hline total $\left(\leqslant 20^{*}\right)$ & 5 & 2 & NS \\
\hline severe $(21-61 *)$ & 13 & 18 & \\
\hline moderate $\left(62-90^{*}\right)$ & 9 & 22 & \\
\hline independent $\left(\geqslant 91^{*}\right)$ & 2 & 1 & \\
\hline \multicolumn{4}{|c|}{ Level of ambulatory activity } \\
\hline bed-confinement & 6 & $2^{\mathrm{a}}$ & NS \\
\hline household & 12 & $25^{\mathrm{a}}$ & \\
\hline community & 11 & 16 & \\
\hline
\end{tabular}

$P$-value by Mantel-Haenszel chi-square test. (*) modified

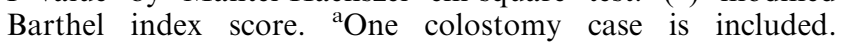
Numbers are cases. NS; not significant care of 23 of the 72 patients in this study corresponded to this definition of 'ideal bowel care of physician', although only 14 of these 23 patients reported no defecation difficulties. On the other hand, among the 49 patients whose bowel care habit did not correspond to the definition of 'ideal bowel care of physician' 29 patients reported no defecation difficulties. If satisfaction with bowel care habits, i.e. 'ideal bowel care of patients', is defined as no defecation difficulties, then the satisfaction rate was $60.8 \%$ in patients who fell within the criteria of 'ideal bowel care of physician' and $59.2 \%$ in those who did not (Table 7). There was, in fact, virtually no differences between two groups, which indicates that for physicians and for patients, the concept of what contributes ideal bowel care is very different.

\section{Discussion}

Spinal cord injury has been found to affect the physiology of the G-I tract; problems include delayed

Table 6 Bowel dysfunction related to frequency, time and method of bowel care

\begin{tabular}{lrrr}
\hline & $\begin{array}{c}\text { Defecation difficulty } \\
\text { Present }\end{array}$ & Absent & P-value \\
\hline Usual method $^{\text {a }}$ & & & \\
$\quad$ Self defecation & 4 & 17 & $P<0.05^{b}$ \\
Anal massage & 11 & 14 & \\
Finger enema & 6 & 7 & \\
Rectal suppository & 8 & 3 & \\
Frequency (once per _days) & 3 & 13 & $P<0.05^{c}$ \\
1 and below & 7 & 12 & \\
$11 / 2$ to 2 & 14 & 13 & \\
3 to 4 & 5 & 5 & \\
5 and over & 2 & 10 & $P<0.05^{c}$ \\
Time for defecation (mins) & & & \\
less than 20 & 9 & 26 & \\
about 30 (21 to 44) & 14 & 7 & \\
about 60 (45 to 60) & 4 & 0 & \\
about 90 (over 61) & 4 & & \\
\hline
\end{tabular}

${ }^{\mathrm{a}}$ Two colostomy cases excluded; ${ }^{\mathrm{b}} P$-value by Chi-square test. ${ }^{\mathrm{c}} P$-value by Mantel-haenszel chi-square test. Numbers are cases

Table 7 Ideal bowel care, Physician vs Patient

\begin{tabular}{lccc}
\hline \multicolumn{3}{c}{$\begin{array}{c}\text { Ideal bowel care of patient } \\
\text { Within }\end{array}$} & $\begin{array}{c}\text { Satisfaction }^{\text {Outside }} \\
\text { rate }^{c}(\%)\end{array}$ \\
\hline $\begin{array}{l}\text { Ideal bowel care of physician } \\
\text { Within criteria }\end{array}$ & 14 & 9 & 60.8 \\
Outside criteria & 29 & 20 & 59.2 \\
\hline
\end{tabular}

${ }^{a}$ Ideal bowel care of physician is defined as spontaneous or reflex defecation without enema or suppository, at least once every 2 days and within $30 \mathrm{~min} .{ }^{\mathrm{b}}$ Ideal bowel care of patient is defined as lack of defecation difficulties. ${ }^{c}$ Percentage of patients with no defecation difficulties 
gastric emptying, altered gastric acid secretion caused by autonomic dysfunction, and abnormal colonic myoenteric activity. ${ }^{2,9}$ In SCI involving cord transection, the G-I reflex remains and defecation using this reflex is commonly recommended. Where there is a combined cauda equina lesion, the pathophysiology is different from that occurring in cord transection patients; in particular the gastrointestinal reflex is absent and anal sphincter tone is reduced; ${ }^{10}$ the method of defecation and the radiological findings are, therefore, also different. ${ }^{11}$ In this study patients with SCI and a combined with cauda equina lesion were excluded because of different pathophysiology, different level of activity, and a lack of diversity in recommended bowel care methods.

Stone et $a l^{12}$ reported that $27 \%$ of SCI patients had significant chronic gastrointestinal problems, and Levi et $\mathrm{al}^{13,14}$ reported gastrointestinal symptoms in $49 \%$ of SCI patients and bowel function problems in $38 \%$. Kannisto and Rintala ${ }^{15}$ studied bowel function in adults who sustained SCI in childhood and reported that although they were better adapted to their bowel function than those who sustained SCI developed in adulthood and encountered only mild problems in their social life, the majority of these patients $(69 \%)$ had bowel dysfunction. In this study the incidence of chronic gastrointestinal problems was also very high $(62.5 \%)$, and in many ways bowel dysfunction was the most serious problem to affect daily activities. In the course of interviews, however, we noticed that compared to the morbidity of this condition, the frequency of medical consultation was relatively low; the attitude of some patients was that bowel dysfunction was their own problem, and they would solve it themselves. Furthermore, decisions relating to the method and frequency of bowel care had been based on the patient's wishes. This suggests both lack of interest on the part of the physician and lack of effective bowel programs.

In studies of factors affecting these G-I problems, Stone et $a l^{12}$ and Gore et $a l^{16}$ reported that the greater the severity of gastrointestinal problems, the higher their incidence; Gulati et $a l^{17}$ reported that prevalence of bowel dysfunction was not related to neurologic level of injury, and to whether or not this was complete. Stone et $a l^{12}$ reported, however, that the prevalence of chronic gastrointestinal problems increased with time after injury, suggesting that these problems were preventable, and Gulati et al ${ }^{17}$ was therefore also pointed that their high incidence was partly due to ineffective bowel programs and partly preventable. Considering our research which showed that chronic G-I problems and bowel dysfunction were not related to severity of neurologic insult, duration of SCI, and level of activity, and most bowel habits had settled within 6 months, we therefore propose that the importance of initiating a bowel program during the early stage of rehabilitation should be recognized.

This study aimed to define an ideal or balanced bowel profile, like a balanced bladder, and an effective bowel program. Because of a lack of research in the field of bowel dysfunction and because of the characteristics of bowel care habit, a matter according to this study is very subjective and individualized, it is hard to define satisfactory bowel care habits. A physician, for example, might define ideal bowel care of physician' as reflex defecation without mechanical aids, performed at least once every 2 days and completed within $30 \mathrm{~min}$; for patients, satisfactory bowel care, that is 'ideal bowel care of patients', corresponded to what, for physicians, was poor. This indicates that ideal bowel care was very subjective and individualized, and for physicians and for patients, the concept of what constitutes ideal bowel care was very different. Not only is the incidence of these problems high, but they also seriously affect daily activity; further study to determine effective methods of bowel care is therefore needed.

\section{Conclusion}

We conclude that (1) the incidence of chronic gastrointestinal problems in SCI patients was very high $(62.5 \%)$, and that most were associated with defecation difficulties; (2) These problems were vague and very subjective, but serious enough to adversely affect various aspects of daily life; (3) Bowel dysfunction was not related to age, duration and neurological level of SCI, ASIA score, or level of activity; (4) With regard to frequency, time and method of defecation, bowel care habits varied considerably among individuals, and in relation to the extent to which practical results matched the levels of expectation generated by a physician's recommended bowel care program, individual satisfaction was also very subjective.

In most SCI patients, bowel care habits were not related to its severity and became settled within 6 months of injury, thus we therefore suggest that during the early stage of rehabilitation, an appropriate bowel program should be carefully designed and appropriate training be provided.

\section{References}

1 Buchanan LE, Nawoczenski DA. Spinal Cord Injury, Concept and Management Approaches. 1st edn, Williams \& Wilkins: Baltimore 1987, pp 93-98.

2 DeLisa JA, Gans BM. Rehabilitation Medicine, Principles and Practice. 2nd edn. J.B. Lippincott Company: Philadelphia 1988, pp 886-915.

3 Bar-On Z, Ohry A. The acute abdomen in spinal cord injury individuals. Paraplegia 1995; 33: $704-706$.

4 Berlly MH, Wilmot CB. Acute abdominal emergencies during the first four weeks after spinal cord injury. Arch Phys Med Rehabil 1984; 65: $687-690$.

5 Leramo OB, Tator $\mathrm{CH}$, Hudson AR. Massive gastrointestinal hemorrhage and perforation in acute spinal cord injury. Surg Neurol 1982; 17: 186-190.

6 Walters K, Silver JR. Gastrointestinal bleeding in patients with acute spinal injuries. Int Rehabil Med 1985; 8: 44-47.

7 Thompson WG, Heaton KW. Functional bowel disorder in apparently healthy people. Gastroenterology 1980; 79: 283-288. 
8 Goodgold J. Rehabilitation Medicine. C.V. Mosby: St Louis 1988, pp 3-25.

9 Aaronson MJ, Freed MM, Burakoff R. Colonic myoelectric activity in persons with spinal cord injury. Dig Dis Sci 1985; 30(4): $295-300$.

10 Banwell JG, Creasey GH, Aggarwal AM, Mortimer T. Management of the neurogenic bowel in patients with spinal cord injury. Urol Clin N Am 1993; 20(3): 517-526.

11 Nino-Murcia M, Friedland GW. Functional abnormalities of the gastrointestinal tract in patients with spinal cord injuries: evaluation with imaging procedures. AJR 1992; 158: $279-281$.

12 Stone JM, Nino-Murcia M, Wolfe VA, Perkash I. Chronic gastrointestinal problems in spinal cord injury patients: a prospective analysis. Am J Gastroenterology 1990; 85: $1114-$ 1119.
13 Levi R, Hulting C, Seiger A. The Stockholm spinal cord injury study: 1. medical problems in a regional SCI population. Paraplegia 1995; 33: $308-315$.

14 Levi R, Hulting C, Seiger A. The Stockholm spinal cord injury study: 2. association between clinical patients characteristics and post-acute medical problems. Paraplegia 1995; 33: 585-594.

15 Kannisto M, Rintala R. Bowel dysfunction in adults who have sustained spinal cord injury in childhood. Paraplegia 1995; 33: $701-703$.

16 Gore RM, Mintzer RA, Calenoff L. Gastrointestinal complications of spinal cord injury. Spine 1981; 6: $538-544$.

17 Gulati MS, Kirshblum SC, Vorman S, O'Connor KC. Bowel care practices in chronic spinal cord injury patients. Arch Phys Med Rehabil 1995; 76: 1040 . 